\title{
A new contact killing toxin permeabilizes cells and belongs to a large protein family
}

2

3

4

5

6

7

8

\author{
Cristian V. Crisan ${ }^{1,2,3}$, Harshini Chandrashekar ${ }^{4}$, Catherine Everly ${ }^{1,2,3}$, Gabi Steinbach ${ }^{3,5}$, \\ Shannon E. Hill2,6, Peter J. Yunker ${ }^{3,5}$, Raquel R. Lieberman ${ }^{2,6}$, Brian K. Hammer ${ }^{1,2,3}$ *
}

${ }^{1}$ School of Biological Sciences, Georgia Institute of Technology, Atlanta, Georgia, USA

${ }^{2}$ Parker H. Petit Institute for Bioengineering \& Bioscience, Georgia Institute of Technology, Atlanta, Georgia, USA

${ }^{3}$ Center for Microbial Diseases and Infection, Georgia Institute of Technology, Atlanta, Georgia, USA

${ }^{4}$ Department of Bioengineering, University of Pennsylvania, Philadelphia, USA

${ }^{5}$ School of Physics, Georgia Institute of Technology, Atlanta, Georgia, USA

${ }^{6}$ School of Chemistry and Biochemistry, Georgia Institute of Technology, Atlanta, Georgia, USA

*Corresponding author: brian.hammer@biology.gatech.edu 9

(1)

21

2

3

4

5

6

7

8

9

0


ABSTRACT

Vibrio cholerae is an aquatic Gram-negative bacterium that causes severe diarrheal cholera disease when ingested by humans. To eliminate competitor cells in both the external environment and inside hosts, $V$. cholerae uses the Type VI Secretion System (T6SS). The T6SS is a macromolecular weapon employed by many Gram-negative bacteria to deliver cytotoxic proteins into adjacent cells. In addition to canonical T6SS gene clusters encoded by all sequenced $V$. cholerae isolates, strain BGT49 encodes an additional locus, which we named auxiliary cluster 4 (Aux 4). The Aux 4 cluster is located on a mobile genetic element and can be used by killer cells to eliminate both $V$. cholerae and Escherichia coli cells in a T6SS-dependent manner. A putative toxin encoded in the cluster, which we name TpeV ( Type VI Permeabilizing Effector Vibrio), shares no homology to known proteins and does not contain motifs or domains indicative of function. Ectopic expression of TpeV in the periplasm of E. coli permeabilizes cells and disrupts the membrane potential. Using confocal microscopy, we confirm that susceptible target cells become permeabilized when competed with killer cells harboring the Aux 4 cluster. We also determine that $t p i V$, the gene located immediately downstream of tpe $V$, encodes an immunity protein that neutralizes the toxicity of TpeV. Finally, we show that $\mathrm{TpeV}$ homologs are broadly distributed across important animal and plant pathogens and are localized in proximity to other T6SS genes. Our results suggest that $\mathrm{TpeV}$ is a toxin that belongs to a large family of T6SS proteins.

\section{IMPORTANCE}

Bacteria live in polymicrobial communities where competition for resources and space is essential for survival. Proteobacteria use the T6SS to eliminate neighboring cells and cause disease. However, the mechanisms by which many T6SS toxins kill or inhibit susceptible target cells are 
54 poorly understood. The sequence of the $\mathrm{TpeV}$ toxin we describe here is unlike any previously described protein. We demonstrate that it has antimicrobial activity by permeabilizing cells, eliminating membrane potentials and causing severe cytotoxicity. TpeV homologs are found near known T6SS genes in human, animal and plant bacterial pathogens, indicating that the toxin is a representative member of a broadly distributed protein family. We propose that TpeV-like toxins contribute to the fitness and pathogenicity of many bacteria. Finally, since antibiotic resistance is a critical global health threat, the discovery of new antimicrobial mechanisms could lead to the development of new treatments against resistant strains.

\section{INTRODUCTION}

The Type VI Secretion System (T6SS) is a common bacterial weapon employed by many killer Gram-negative bacteria to translocate toxic protein effectors into adjacent target cells $(1,2)$. The harpoon-like proteinaceous apparatus is anchored to the membrane of killer cells by the membrane complex, which spans the inner membrane and periplasm (3-5). Hcp (hemolysin-coregulated protein) hexamers stack to form an inner tube that is capped at the distal end by a trimer of VgrG (valine-glycine repeat protein $\mathrm{G}$ ) tip-forming proteins $(2,6,7)$. PAAR (proline-alanine-alaninearginine) proteins also interact with VgrGs and expand the toxin repertoire $(8,9)$. The T6SS uses a contraction mechanism that propels the inner tube and delivers the toxic payload (10-12).

Vibrio cholerae is a wide-spread gastrointestinal pathogen that has caused seven cholera pandemics (13). The bacterium is found in polymicrobial marine ecosystems in association with copepods, fish and insects (14-16). To colonize hosts and survive in environmental settings, $V$. cholerae employs T6SS effectors that disrupt the cell envelope of competitor cells (17-24). T6SS genes are distributed across a large cluster and two auxiliary clusters in all sequenced $V$. cholerae isolates $(25,26)$. In clinical strains like V52 and C6706, the large gene cluster encodes a VgrG tip- 
77 forming protein with a C-terminal peptidoglycan-degrading domain (23). Auxiliary clusters 1 and 782 encode the TseL lipase and VasX colicin-like effectors, respectively (19-22). An auxiliary 79 cluster 3 is found in a subset of $V$. cholerae isolates and contains a peptidoglycan-degrading toxin $80(27-29)$.

81 Although most clinical $V$. cholerae strains encode T6SS effectors with conserved activities, $V$. 82 cholerae strains obtained from sources other than patients harbor a more diverse repertoire of T6SS 83 toxins $(25,26,30,31)$. We previously identified auxiliary 5 (Aux 5) T6SS clusters in several $V$. 84 cholerae strains, which encode predicted phospholipase effectors (25). Recently, several $V$. cholerae strains have been shown to possess an Aux 6 T6SS cluster with antibacterial activity (31).

86 We and others have also reported that many $V$. cholerae strains (but not C6706) contain an 87 additional gene cluster with putative T6SS components, which we named Aux 4 (25, 32). 88 However, the activity of the cluster, the roles played by the encoded genes in microbial competition and the toxicity of the putative effector have not been validated. Here we demonstrate that the Aux 4 cluster can be used by $V$. cholerae to kill bacterial cells in a T6SS-dependent manner. We report that the toxin found within the cluster permeabilizes cells and

92 disrupts the membrane potentials when expressed in the periplasm of Escherichia coli cells. A 93 protein encoded by a gene found immediately downstream of the effector neutralizes its toxicity 94 and acts as a protective immunity factor. Finally, we show that homologs of the Aux 4 effector are 95 found in diverse bacterial species, including human, animal and plant pathogens. The potent 96 antimicrobial activity of $\mathrm{TpeV}$ and broad distribution of identified homologs suggest the toxins 97 confer significant competition advantages to bacteria that harbor them. 


\section{RESULTS}

100

101

102

103

104

105

106

107

108

109

110

111

112

113

114

115

116

117

118

The Aux 4 tpeV-tpiV are an active effector-immunity pair in strain BGT49

$V$. cholerae strain BGT49 encodes the Aux 4 cluster, in addition to the canonical T6SS large operon and auxiliary clusters 1 and 2 (Fig. 1A). The Aux 4 cluster contains predicted T6SS genes: an hcp, a vgrG, a DUF4123 chaperone, and a paar (33) (Fig. 1A). Genes coding for a putative

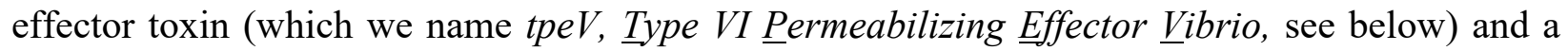
putative immunity protein (which we name tpiV, Type VI Permeabilizing Immunity $\underline{\text { Vibrio, }}$, see below) are also found within the cluster (Fig. 1A) $(32,34,35)$. The $\operatorname{vgr} G$ gene does not contain a toxic C-terminal domain, as described for the $V$. cholerae VgrG-1 or VgrG-3 $(23,36,37)$.

Genes for a restriction modification system are found upstream of the Aux 4 cluster (Fig. 1A). Both the Aux 4 T6SS cluster and restriction modification system genes are flanked upstream by a predicted integrase and downstream by a predicted transposase (Fig. 1A). Attachment (att) sites similar to those found in the Vibrio pathogenicity island 1 (VPI-1) also flank the region $(32,38)$.

To experimentally determine that the tpe $V$ gene encodes a T6SS toxin, we engineered a $\Delta t p e V \Delta t p i V$ target BGT49 strain. The $\Delta t p e V \Delta t p i V$ target strain was then co-cultured with either wild type BGT49, AtpeV (BGT49 lacking the TpeV effector) or BGT49 T6SS- killers. The recovery of the $\Delta t p e V \Delta t p i V$ target strain was significantly reduced (by approximately 5 orders of magnitude) when co-cultured with wild type BGT49 killer cells compared to the 4 tpeV or T6SSkiller strains (Fig. 1B). This result indicates that TpeV is a T6SS effector that is actively used by $V$. cholerae strain BGT49 to eliminate susceptible cells that lack the TpiV immunity protein. 
Aux 4

$\begin{array}{lll}\text { A) } & \square \text { YafY transcriptional regulator } \square \text { RadC DNA Repair } \\ \square \text { Prophage integrase IntA } & \square \text { DUF2787 } \\ \square \text { HdsM restriction methyltransferase } & \square \text { Hcp } & \square \text { DUF2787 } \\ \square \text { HNH endonuclease } & \square \text { DUF4123 } & \square \text { YeeP GTPase } \\ \square \text { AAA ATPase } & \square \text { tpeV } & \square \text { Transposase } \\ \square \text { Type I restriction enzyme } & \square \text { tpiV } & \\ \square \text { HdsR restriction endonuclease } & \square \text { paar } \\ \square \text { Unknown } & \square \text { DUF2628 } \\ \square \text { SbcC DNA repair exonuclease } & \square \text { Unknown } \\ \square \text { DUF4194 } & \square \text { AlpA transcriptional regulator }\end{array}$

B)

Target: $V$. cholerae BGT49 $\Delta$ tpeVAtpiV

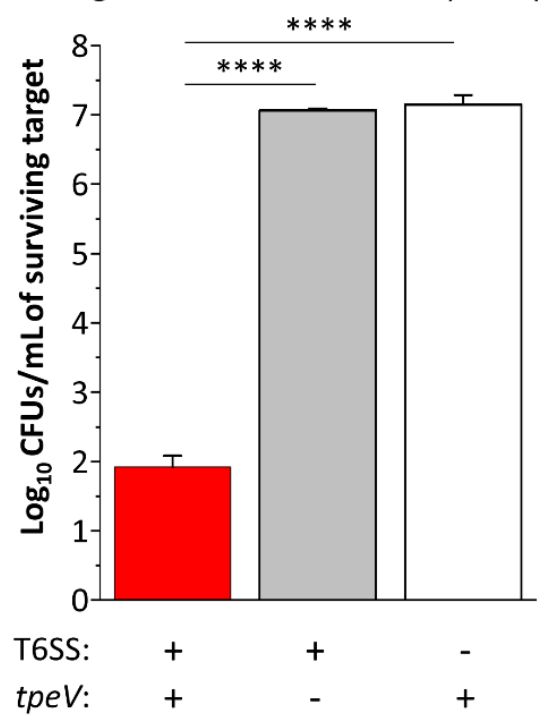

Killer V. cholerae BGT49 genotypes

121 Figure 1. Vibrio cholerae strain BGT49 encodes the Aux 4 T6SS cluster and efficiently 122 eliminates target bacteria in a TpeV and T6SS-dependent manner. A) The Aux 4 cluster 123 encodes predicted $h c p$, vgrG, DUF4123-containing chaperone, effector, immunity and paar genes.

124 The cluster is found on a predicted mobile genetic element, being flanked by integrase and 125 transposase genes. B) Target $V$. cholerae BGT49 $\Delta t p e V \Delta t p i V$ was co-cultured with either WT, $126 \Delta t p e V$ or T6SS- killer BGT49. A one-way ANOVA with a post hoc Tukey HSD test was used to 127 determine significance. $* * * * \mathrm{p}<0.0001$ 
The Aux 4 cluster can be transferred to another $V$. cholerae strain where it confers competitive advantages

Since we observed that the Aux 4 cluster is located on a predicted mobile genetic element, we hypothesized that it can be used by other $V$. cholerae strains to eliminate competitor cells in a T6SS-dependent manner. In $V$. cholerae C6706, the QstR protein is a gene regulator that is required and sufficient to induce expression of T6SS genes $(39,41)$. We cloned the Aux4 vgrG, tap, tpeV, tpiV and paar genes on a plasmid (pAux4) under control of the Ptac promoter. We then introduced the pAux4 plasmid in $V$. cholerae strain C6706*, which constitutively expresses the QstR protein but does not possess Aux 4 cluster genes on its chromosomes $(24,39-41)$. The $V$. cholerae C6706* killer with the Aux 4 cluster on a plasmid (C6706*/pAux4) efficiently eliminates the parental target strain, unlike a killer C6706* strain carrying a plasmid control (Fig. 2A). By contrast, a C6706*/pAux4 T6SS- strain cannot eliminate the parental target strain (Fig. 2A). To provide further evidence that TpiV can confer immunity, we introduced the tpiV gene into target V. cholerae C6706 and co-cultured the strain with killer C6706*/pAux4 cells. V. cholerae C6706*/pAux4 kills $V$. cholerae target cells with a plasmid control, but not when they encode the tpiV gene (Fig. 2B).

We next inquired if the Aux 4 cluster can be used by $V$. cholerae to kill other target bacterial species. A C6706* strain with a functional T6SS that lacks both native TseL and VasX effectors poorly eliminates E. coli cells compared to a C6706* strain that harbors both toxins (Fig. 2C) (42, 43). However, the introduction of the Aux 4 cluster into the C6706* strain lacking TseL and VasX effectors restores its ability to efficiently eliminate $E$. coli cells (Fig. 2C). We recently reported that target E. coli cells are protected against T6SS attacks from strain C6706* when co-cultured on LB medium supplemented with $0.4 \%$ glucose (44). By contrast, we observe that killer 
$151 \mathrm{C} 6706 * / \mathrm{pAux} 4$ can bypass the glucose-mediated resistance to efficiently eliminate $E$. coli cells

152 even when the co-culture is performed on LB medium with glucose (Fig. 2D). These results

153 confirm that the Aux 4 intoxicates competitor bacterial cells.

A)

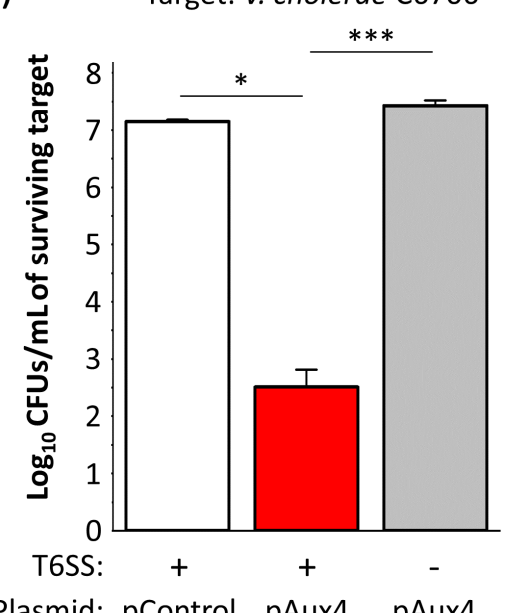

Plasmid: pControl pAux4 pAux4

Killer V. cholerae C6706* genotypes

C)

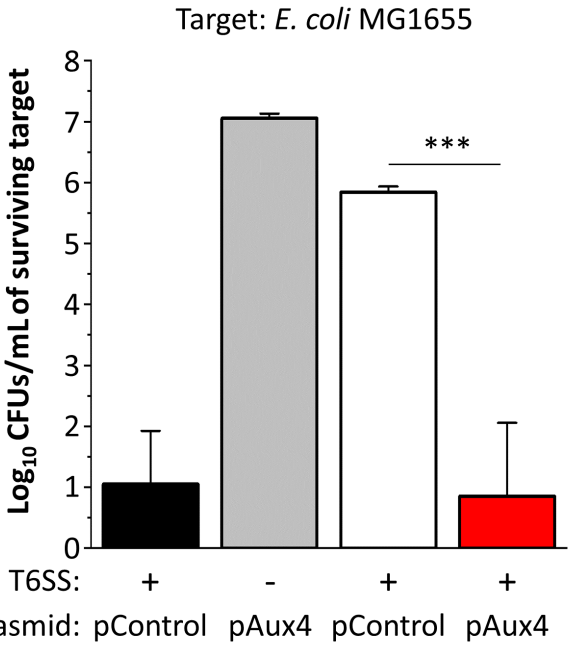

TseL and VasX: $\quad+\quad+\quad-\quad-$

Killer V. cholerae C6706* genotypes
B) Killer: V. cholerae $66706 * /$ pAux4

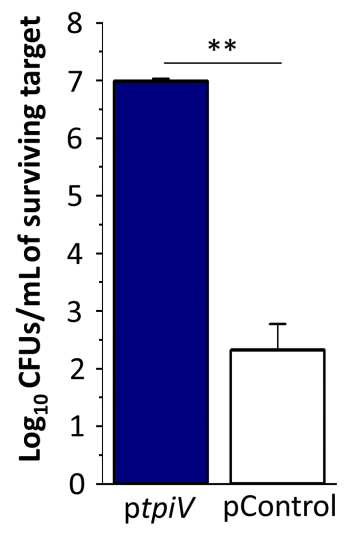

Target V. cholerae C6706* genotypes
D)

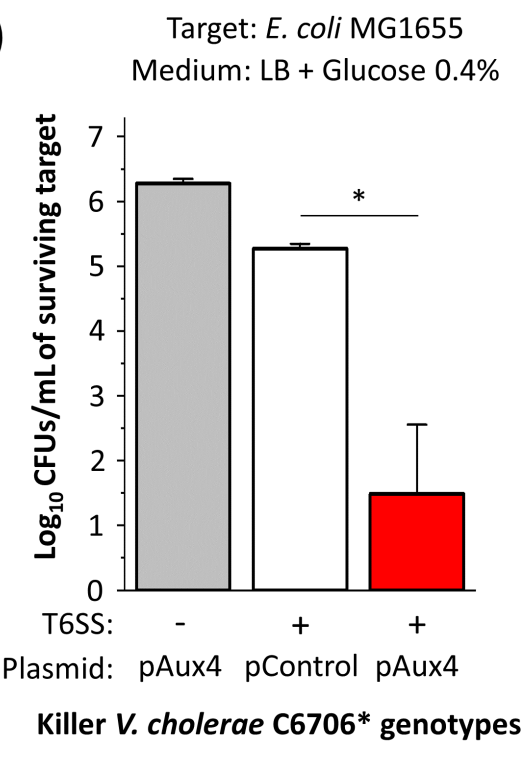

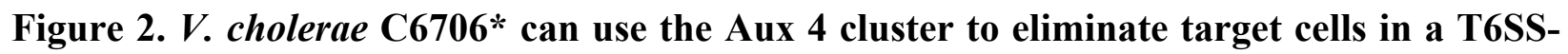
dependent manner. A) $V$. cholerae $66706^{*}$ (T6SS+ or T6SS-) with a plasmid control or a plasmid encoding the Aux 4 cluster were co-cultured with target parental $V$. cholerae C6706. A one-way ANOVA with a post hoc Tukey HSD test was used to determine significance. B) Killer V. cholerae C6706* with the Aux 4 cluster were co-cultured with target C6706 cells with a plasmid control or 
a plasmid encoding tpi $V$. Welch's t-test was used to determine significance. C) $V$. cholerae C6706* with deletions in the known tseL and vasX T6SS effectors containing either a plasmid control or a plasmid with Aux 4 was co-cultured with E. coli MG1655 cells. A one-way ANOVA with a post hoc Tukey HSD test was used to determine significance. D) $V$. cholerae C6706* with a plasmid control or a plasmid encoding the Aux 4 cluster were co-cultured with E. coli MG1655 on LB medium with $0.4 \%$ glucose. A one-way ANOVA with a post hoc Tukey HSD test was used to determine significance. $* * * \mathrm{p}<0.001, * * \mathrm{p}<0.01 * \mathrm{p}<0.05$

\section{TpeV permeabilizes cells and disrupts the membrane potential}

We next used confocal microscopy to examine co-cultures between fluorescently labelled target V. cholerae C6706 cells (shown as cyan) and unlabeled killer C6706*/pAux4 cells (Fig. 3A). To each co-culture we added propidium iodine (PI), a molecule that cannot penetrate cells with intact membranes but exhibits high fluorescence when bound to the DNA of cells with compromised membranes. Fluorescently labelled $V$. cholerae target cells are successfully eliminated when cocultured with killer C6706*/pAux4 cells but remain viable when killer cells cannot assemble the T6SS apparatus (T6SS-) (Fig. 3A). Furthermore, a robust PI signal (depicted with red) is detectable when target cells are co-cultured with C6706*/pAux4 cells (Fig. 3A).

Since we observed that $V$. cholerae cells harboring the Aux 4 cluster can kill and permeabilize target cells in a T6SS-depedent manner, we sought to further characterize the activity of the TpeV effector. The protein does not share primary sequence homology to known toxins and does not contain motifs or domains indicative of function. Tertiary structural prediction algorithms also fail to detect significant homologs with known functions. TpeV has 11 cysteine residues, suggesting that multiple disulfide bonds could play roles in stabilizing the protein. Transmembrane prediction algorithms TMHMM and Phobius do not detect extensive transmembrane regions and SignalP 5.0

does not predict a signal sequence (Supplementary Fig. 1 and 2) (45-47). We also attempted to identify TpeV homologs using the secondary structure predictor Jpred (48). While most homologs 
are hypothetical proteins with unknown functions, some contain domains similar to the peptidoglycan-binding C-terminal regions of the OmpA protein $(49,50)$. OmpA proteins are involved in pathogenesis and have diverse functions that include formation of porins and channels $(51,52)$. Because target $V$. cholerae cells have a substantial PI signal when co-cultured with killer cells harboring the Aux 4 cluster, we hypothesized that $\mathrm{TpeV}$ might permeabilize target cells when delivered to the periplasm.

To test this prediction, we introduced plasmid-borne tpeV with a periplasmically-directing $\mathrm{pel} B$ sequence under the control of an inducible promoter into E. coli cells. A significantly higher PI signal is detected when E. coli cells are induced to express periplasmic TpeV compared to cells that harbor a plasmid control (Fig. 3B). We also hypothesized that TpeV disrupts the bacterial cell membrane potential $(19,53,54)$. To test this hypothesis, we used the Bis-(1,3-Dibutylbarbituric acid) Trimethine Oxonol (( $\left.\mathrm{DiBAC}_{4}(3)\right)$ potential-sensitive dye, which is excluded from cells with a normal membrane potential but exhibits fluorescence in depolarized cells (54-56). E. coli cells that express periplasmically-delivered $\mathrm{TpeV}$ have significantly higher $\mathrm{DiBAC}_{4}(3)$ uptake compared to E. coli that express a plasmid control (Fig. 3C). To probe if $\mathrm{TpeV}$ can disrupt the membrane potential in a T6SS-dependent manner, we co-cultured $V$. cholerae BGT49 $\Delta$ tpeVAtpiV target cells with either BGT49 wild type or BGT49 $\Delta t p e V$ killers. Following co-cultures with wild type but not BGT49 $\Delta$ tpeV killers, bacterial membrane potentials are disrupted as cells display an elevated $\mathrm{DiBAC}_{4}(3)$ signal (Fig. 3C). Taken together, these findings demonstrate that $\mathrm{TpeV}$ permeabilizes cells and disrupts the membrane potential of target bacteria. 


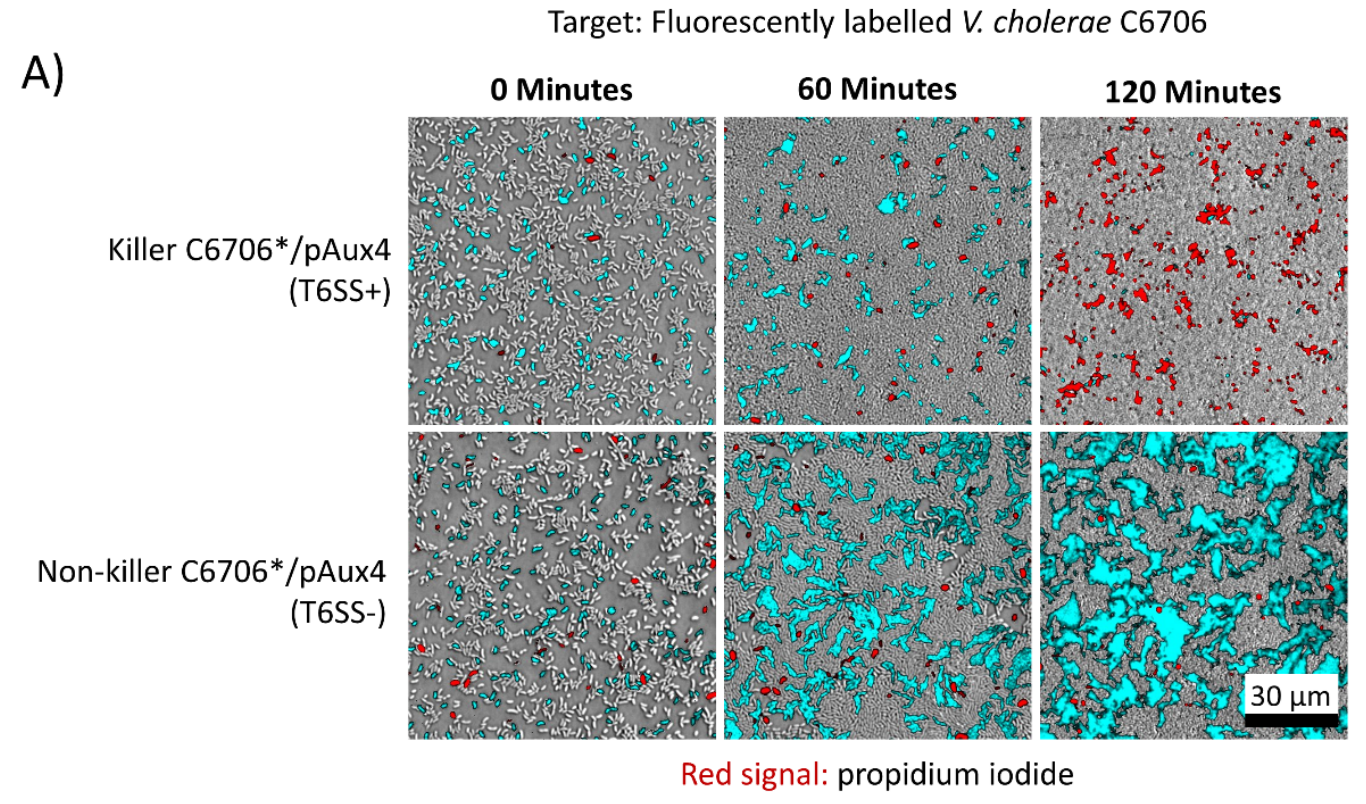

Target: Fluorescently labelled V. cholerae C6706

Red signal: propidium iodide

B)

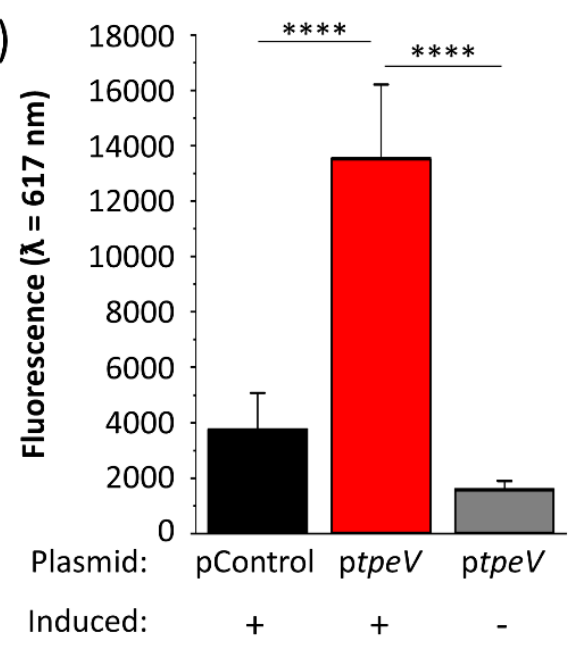

\section{E. coli genotypes and treatments}

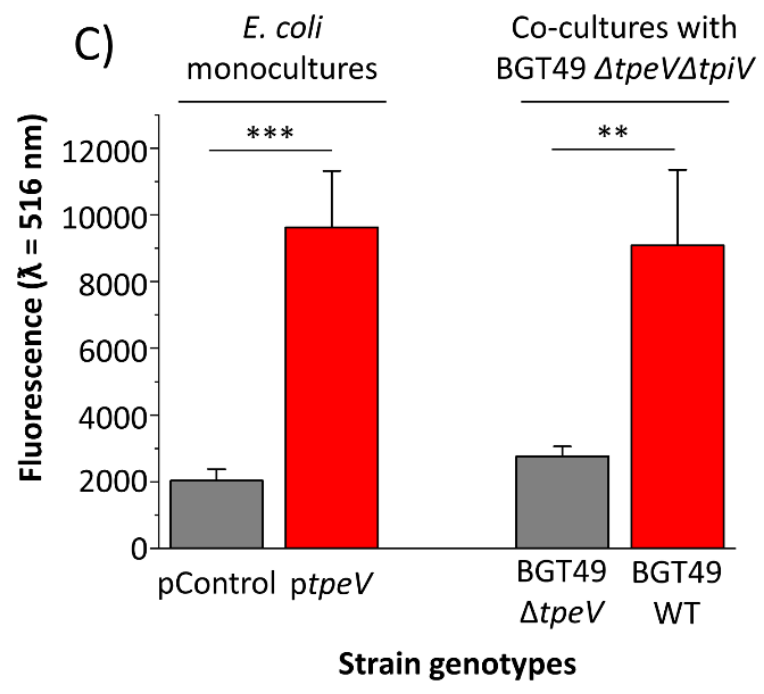

Figure 3. TpeV permeabilizes target cells and disrupts the membrane potential, leading to cytotoxicity. A) Confocal microscopy was used to visualize a co-culture between C6706* cells with Aux 4 (T6SS- or T6SS+) and fluorescently labelled target C6706 cells in the presence of propidium iodide. Scale bar $=30 \mu \mathrm{m}$. B) E. coli cells carrying a periplasmic tpeV construct or plasmid control were incubated with propidium iodide. Fluorescence readings were taken at an excitation $\chi=535 \mathrm{~nm}$ and emission $\chi=617 \mathrm{~nm}$. A one-way ANOVA with a post hoc Tukey HSD test was used to determine significance. C) E. coli cells carrying a periplasmic tpeV construct or plasmid control, or $V$. cholerae BGT49 co-cultures between target $\Delta t p e V \Delta t p i V$ and wild type or $\Delta$ tpe $V$ killer cells were incubated with the membrane potential-sensitive $\operatorname{DiBAC}_{4}(3)$ dye. Fluorescence readings were taken at an excitation $\chi=490 \mathrm{~nm}$ and emission $\chi=516 \mathrm{~nm}$. Welch's t-tests were used to determine significance. $* * * * p<0.0001, * * * \mathrm{p}<0.001, * * \mathrm{p}<0.01$ 
Since the sequence or predicted structure of TpeV shares no homology to known toxins (including competitor bacteria.

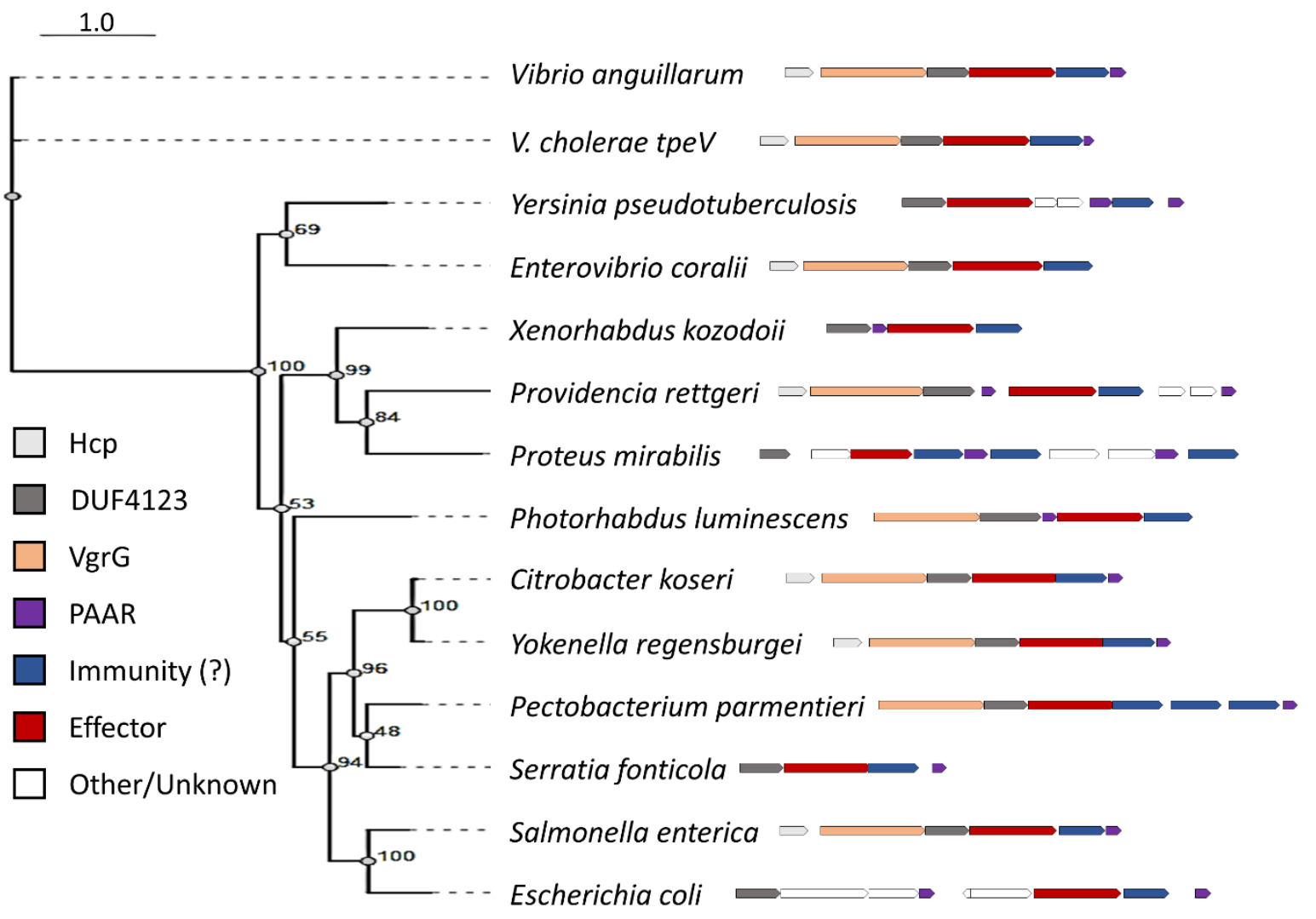




\section{DISCUSSION}

248

249

250

251

252

253

254

255

256

257

258

259

260

261

262

263

264

265

266

267

268

269

Here we show that many bacterial species encode homologs of a previously undescribed T6SS protein that intoxicates, permeabilizes and disrupts the membrane potential of target cells. While studies have examined antibacterial effectors from clinical $V$. cholerae isolates such as C6706 and V52, we and others found that strains isolated from sources other than patients encode a more diverse set of putative T6SS toxins $(3,19,22,25,26,32)$. The VasX $V$. cholerae effector encoded in the Aux 2 cluster is a large protein that contains a C-terminal colicin domain effective at eliminating both bacterial and eukaryotic cells $(19,20)$. Since it is predicted to form large pores, VasX permeabilizes cells and allows passage of molecules like PI into the cell (19). The Pseudomonas aeruginosa Tse4 and the Serratia marcecens Ssp6 effectors are both relatively small proteins that form ion-selective pores but do not allow larger molecules like PI to enter cells (53, 54). Recently, Vibrio parahaemolyticus has also been shown to harbor T6SS effectors that disrupt cellular membranes (58).

Importantly, the $V$. cholerae TpeV T6SS effector we describe in this study does not contain predicted domains or motifs with known functions and its sequence does not share homology to any previously described T6SS effectors. We provide evidence that TpeV is a T6SS toxin that can be used by $V$. cholerae cells to permeabilize target cells and disrupt the cell membrane potential (Fig. 2, Fig. 3). The cell membrane potential is essential for ATP synthesis, cell division and membrane transport (59-61). Therefore, TpeV-mediated toxicity is likely to inflict substantial damage to target cells by perturbing multiple essential processes.

We hypothesize that TpeV could permeabilize cells by forming pores. Pore-forming toxins (PFTs) are widespread among all kingdoms of life (62-66). Based on the secondary structure of the membrane spanning domain, two major classes of pore-forming toxins (PFTs) have been 
described: $\alpha$-PFTs and $\beta$-PFTs $(62,64,67), \alpha$-PTFs include the $E$. coli colicin and cytolysin A families, while $\beta$-PFTs are found in many Gram-positive bacterial species and contribute to the virulence of pathogens like Staphylococcus aureus and Clostridium perfringens $(64,66-68)$. Our homology predictions suggest that $\mathrm{TpeV}$ might harbor a peptidoglycan-binding OmpA-like domain (49). RmpM is a Neisseria meningitidis periplasmic protein that also possesses an OmpAlike domain (50). Experimental evidence indicates that RmpM stabilizes oligomeric porins in the outer membrane $(50,69)$. Rather than form new pores, it is also possible that TpeV might interact with and disrupt the normal functions of existing porins or channels in the membranes of target bacteria. Future experiments will determine if $\mathrm{TpeV}$ forms pores or employs other mechanisms that damage membranes and permeabilize cells.

In strain BGT49, the Aux 4 cluster and a restriction modification system are found near a prophage integrase and a transposase (Fig. 1A). This suggests that the genes are located on a mobile genetic element that can be transferred between bacterial cells to confer competitive advantages against phages and other bacterial cells (32). The Vibrio cholerae T6SS Aux 3 cluster was also recently shown to be located on a mobile genetic element (29). Our results showing that $V$. cholerae strain C6706* can use the Aux 4 cluster to kill parental cells supports the hypothesis that the Aux 4 cluster can be transferred to confer competitive advantages. This hypothesis is further supported by our observation that $\mathrm{TpeV}$ homologs are found close to other T6SS genes in many bacterial species, including human pathogens (Providencia rettgeri, Proteus mirabilis, Citrobacter koseri, Yokenella regensburgei, Serratia fonticola, Salmonella enterica, and E. coli), animal pathogens (Vibrio anguillarum and Photorhabdus luminescens) and plant pathogens (Pectobacterium parmentieri) (70-77) (Fig. 4). TpeV homologs found in other bacterial species are also located near transposase-like genes (data not shown). 
293

294

295

296

297

298

299

300

301

302

303

304

305

306

307

308

309

310

311

312

313

All known T6SS toxic effectors are neutralized by cognate immunity proteins, which are generally encoded by genes adjacent to effectors $(19,78)$. We found that $t p i V$, the gene found immediately downstream of tpeV, confers immunity to target cells against tpe $V$-mediated toxicity (Fig. $1 \mathrm{~B}$ and 2B). SignalP predicts that TpiV encodes a periplasmic Sec-tag, which is expected since TpeV exhibits its toxicity when delivered to the periplasm of target cells (Supp. Fig. 2, Fig. 3A). In other species, we observed that multiple putative immunity proteins can be found near TpeV homologs (Fig. 4). Additional studies are required to confirm which predicted TpiV-like proteins are the cognate immunity factors for the TpeV homologs.

In conclusion, we demonstrate that the T6SS Aux 4 cluster found in many $V$. cholerae isolates encodes a toxin that can be used to eliminate competitor bacteria. TpeV is a T6SS effector that permeabilizes target bacteria and disrupts the membrane potential, leading to severe cellular intoxication. However, target cells expressing TpiV are protected and resist TpeV-mediated toxicity. Finally, we find that $\mathrm{TpeV}$ homologs are widespread among Gram-negative bacteria, suggesting the protein represents a novel and potent antimicrobial agent of interest for further studies. Understanding the molecular mechanisms of antimicrobial toxins that drive competition and antagonism could lead to the development of novel biotechnology and medical applications. 


\section{METHODS}

315

316

317

318

320

321

322

323

324

325

326

327

328

329

\section{Bacterial strains and plasmids}

Plasmids were constructed using standard molecular biology techniques. Gibson mix reagents, restriction enzymes and polymerase were used as recommended by manufacturers (Promega and New England Biolabs). Plasmids were verified by PCR and Sanger sequencing (Eurofins). $V$. cholerae C6706 mutant strains were made using pKAS allelic exchange methods, as described previously (79). V. cholerae strain BGT49 mutant strains were made using natural transformation, as described previously with modifications $(80,81)$. Briefly, overnight cultures were back-diluted in fresh LB medium for approximately 1 hour and then statically incubated overnight at $30^{\circ} \mathrm{C}$ in liquid LB medium with a sterile crab shell fragment. Crab shells were transferred and incubated in fresh LB medium containing $30-50 \mu \mathrm{g}$ of a plasmid engineered to encode $\sim 1000 \mathrm{bp}$ flanking regions to replace the desired genes with an antibiotic cassette. Cells were incubated statically overnight at $30^{\circ} \mathrm{C}$ liquid $\mathrm{LB}$ medium and then spread on antibiotic plates to select for transformants. BGT49 mutants were confirmed by PCR and antibiotic resistance. Bacterial strains and plasmids used are listed in Supplementary Table 2.

\section{Bacterial Competition Assays}

Bacterial cultures were grown overnight in liquid LB medium at $37^{\circ} \mathrm{C}$ with shaking. Overnight cultures were back-diluted and incubated in liquid LB medium at $37^{\circ} \mathrm{C}$ with shaking for 3 hours. Bacterial cultures were then normalized to an $\mathrm{OD}_{600}$ absorbance of 1 . If strains harbored plasmids, cultures were grown overnight with antibiotics to maintain plasmids and $100 \mu \mathrm{M}$ IPTG if plasmids contained an inducible promoter. If strains were grown in media containing antibiotics, liquid cultures were then washed three times with fresh LB medium before they were co-cultured. A 50 
$336 \mu \mathrm{L}$ mixture aliquot of ratio of 10:1 killer:target cells was spotted on a $0.22 \mu \mathrm{m}$ pore size filter

337 paper, which was placed on LB agar media and incubated at $37^{\circ} \mathrm{C}$. After 3 hours, filters were

338 vortexed in sterile LB media for 30 seconds. $100 \mu \mathrm{L}$ of serial dilutions were then spread on plates

339 containing the required antibiotic to select for target cells. Data from three co-cultures were used

340 to determine significance. Results are representative of at least two independent experiments.

341 Confocal microscopy

342 Overnight cultures were back-diluted 1:100 for 3 hours in liquid LB medium. Samples were then

343 normalized to an $\mathrm{OD}_{600}$ of $10.1 \mu \mathrm{L}$ aliquot of 10:1 killer:target cell mixture was spotted on top of

344 a dry $8-\mu \mathrm{L}$ aliquot of propidium iodide $(100 \mu \mathrm{g} / \mathrm{mL})$ on an LB agar pad. Nikon A1R confocal

345 microscope using a Perfect Focus System with a 40x objective (Plan Fluor ELWD 40x DIC M N1)

346 was used to stabilize the focus in the plane of the colony growth. Cells were imaged at 96-100\%

347 humidity and $37^{\circ} \mathrm{C}$. Images were processed using ImageJ. Results are representative of at least

348 three independent experiments.

Membrane permeabilization assays

350 Bacterial cultures of E. coli Shuffle T7 Express (New England Biolabs) cells carrying either a

351 control plasmid or a periplasmic tpeV construct were grown overnight in liquid LB medium

352 supplemented with $0.2 \%$ glucose and ampicillin at $37^{\circ} \mathrm{C}$ with shaking. Cells were washed three 353 times with LB and 100x back-dilutions were made in fresh liquid LB medium with $500 \mu \mathrm{M}$ IPTG 354 and ampicillin. Strains were incubated at $37^{\circ} \mathrm{C}$ for 2 hours, washed three times with PBS and 355 normalized to an $\mathrm{OD}_{600}$ of $1.100 \mu \mathrm{L}$ of each culture was incubated with $1 \mu \mathrm{L}$ propidium iodide $356(100 \mu \mathrm{g} / \mathrm{mL})$ for $15-30$ minutes. Fluorescence values were taken on a Synergy BioTek plate reader 357 using an excitation $\chi=535 \mathrm{~nm}$ and emission $\chi=617 \mathrm{~nm}$ and normalized by subtracting the average 
358 values from samples with propidium iodide but no cells. Data represents the averages obtained 359 from seven biological replicates from two independent experiments.

360

361

362

363

364

365

366

367

368

369

370

371

372

373

374

375

376

377

378

379

\section{Membrane potential assays}

Bacterial cultures of E. coli Shuffle T7 Express (New England Biolabs) cells carrying either a control plasmid or a periplasmic tpeV construct were grown overnight with shaking at $37^{\circ} \mathrm{C}$ in liquid LB medium supplemented with $0.2 \%$ glucose and ampicillin. Cells were washed three times with $\mathrm{LB}$ and $100 \mathrm{x}$ back-dilutions were incubated at $37^{\circ} \mathrm{C}$ for 2 hours in fresh liquid $\mathrm{LB}$ medium with $500 \mu \mathrm{M}$ IPTG and ampicillin. Cells were again washed three times with PBS and normalized to an $\mathrm{OD}_{600}$ of 1 in PBS. Cells were incubated for 30 minutes in the dark with DiBAC4(3) at a final concentration of $10 \mu \mathrm{M}$ and washed with three times with PBS. Fluorescence values were taken on a Synergy BioTek plate reader using an excitation $\chi=490 \mathrm{~nm}$ and emission $\chi=516 \mathrm{~nm}$. Data represents the averages obtained from six biological replicates from two independent experiments.

For co-culture measurements of membrane potentials, overnight cultures of the indicated $V$. cholerae BGT49 strains were back-diluted and incubated in liquid LB medium at $37^{\circ} \mathrm{C}$ with shaking for 3 hours. Bacterial cultures were then normalized to an OD600 absorbance of 1. A 50 $\mu \mathrm{L}$ mixture aliquot of ratio of 1:1 killer:target cells was spotted on a $0.22 \mu \mathrm{m}$ pore size filter paper, which was placed on LB agar media and incubated at $37^{\circ} \mathrm{C}$. After 3 hours, filters were vortexed in sterile LB media for 30 seconds. Cells were washed three times with PBS, normalized to an $\mathrm{OD}_{600}$ of 1 , incubated for 30 minutes in the dark with DiBAC4(3) at a final concentration of $10 \mu \mathrm{M}$ and washed with three times with PBS. Fluorescence values were taken on a Synergy BioTek plate reader using an excitation $\chi=490 \mathrm{~nm}$ and emission $\chi=516 \mathrm{~nm}$. Data represents the averages obtained from six biological replicates from two independent experiments. 
The HHMER server was used to search for homologs of TpeV in the UniProtKB database (82).

Selected homologs were aligned using MUSCLE (83). A phylogenetic tree was constructed using

proteins were predicted based on homology to TpiV and genomic location. Truncated VgrG-like genes with stop codons were observed in some species but were excluded from Fig. 4.

\section{REFERENCES}

1. Pukatzki S, Ma AT, Sturtevant D, Krastins B, Sarracino D, Nelson WC, Heidelberg JF, Mekalanos JJ. 2006. Identification of a conserved bacterial protein secretion system in Vibrio cholerae using the Dictyostelium host model system. Proc Natl Acad Sci 103:1528-1533.

2. Mougous JD, Cuff ME, Raunser S, Shen A, Zhou M, Gifford CA, Goodman AL, Joachimiak G, Ordoñez CL, Lory S, Walz T, Joachimiak A, Mekalanos JJ. 2006. A Virulence Locus of Pseudomonas aeruginosa Encodes a Protein Secretion Apparatus. Science 312:1526-1530.

3. Zheng J, Ho B, Mekalanos JJ. 2011. Genetic Analysis of Anti-Amoebae and AntiBacterial Activities of the Type VI Secretion System in Vibrio cholerae. PLoS One 6:e23876.

4. Aschtgen MS, Thomas MS, Cascales E. 2010. Anchoring the type VI secretion system to the peptidoglycan: TssL, TagL, TagP... what else? Virulence 1:535-540.

5. Rapisarda C, Cherrak Y, Kooger R, Schmidt V, Pellarin R, Logger L, Cascales E, Pilhofer M, Durand E, Fronzes R. 2019. In situ and high-resolution cryo- EM structure of a bacterial type VI secretion system membrane complex . EMBO J 38:1-18.

6. Ballister ER, Lai AH, Zuckermann RN, Cheng Y, Mougous JD. 2008. In vitro selfassembly of tailorable nanotubes from a simple protein building block. Proc Natl Acad Sci 105:3733-3738.

7. Silverman JM, Agnello DM, Zheng H, Andrews BT, Li M, Catalano CE, Gonen T, Mougous JD. 2013. Haemolysin Coregulated Protein Is an Exported Receptor and Chaperone of Type VI Secretion Substrates. Mol Cell 51:584-593.

8. Cianfanelli FR, Alcoforado Diniz J, Guo M, De Cesare V, Trost M, Coulthurst SJ. 2016. VgrG and PAAR Proteins Define Distinct Versions of a Functional Type VI Secretion System. PLoS Pathog 12:e1005735.

9. Wood TE, Howard SA, Wettstadt S, Filloux A. 2019. PAAR proteins act as the 'sorting 
hat' of the type VI secretion system. Microbiology 165:1203-1218.

414

415

416

417

418

419

420

421

422

423

424

425

426

427

428

429

430

431

432

433

434

435

436

437

438

439

440

441

442

443

444

445

446

447

448

449

450

10. Bröms JE, Ishikawa T, Wai SN, Sjöstedt A. 2013. A functional VipA-VipB interaction is required for the type VI secretion system activity of Vibrio cholerae O1 strain A1552. BMC Microbiol 13:96.

11. Bönemann G, Pietrosiuk A, Diemand A, Zentgraf H, Mogk A. 2009. Remodelling of VipA/VipB tubules by ClpV-mediated threading is crucial for type VI protein secretion. EMBO J 28:315-325.

12. Kube S, Kapitein N, Zimniak T, Herzog F, Mogk A, Wendler P. 2014. Structure of the VipA/B Type VI Secretion Complex Suggests a Contraction-State-Specific Recycling Mechanism. Cell Rep 8:20-30.

13. Sack DA, Sack RB, Nair GB, Siddique A. 2004. Cholera. Lancet 363:223-233.

14. de Magny GC, Mozumder PK, Grim CJ, Hasan NA, Naser MN, Alam M, Sack RB, Huq A, Colwell RR. 2011. Role of zooplankton diversity in Vibrio cholerae population dynamics and in the incidence of cholera in the Bangladesh sundarbans. Appl Environ Microbiol 77:6125-6132.

15. Senderovich Y, Izhaki I, Halpern M. 2010. Fish as reservoirs and vectors of Vibrio cholerae. PLoS One 5:e8607.

16. Sela R, Hammer BK, Halpern M. 2020. Quorum-sensing signaling by chironomid egg masses' microbiota, affect Haemagglutinin/Protease (HAP) production by Vibrio cholerae. Mol Ecol 1-11.

17. Logan SL, Thomas J, Yan J, Baker RP, Shields DS, Xavier JB, Hammer BK, Parthasarathy R. 2018. The Vibrio cholerae type VI secretion system can modulate host intestinal mechanics to displace gut bacterial symbionts. Proc Natl Acad Sci 115:E3779E3787.

18. Zhao W, Caro F, Robins W, Mekalanos JJ. 2018. Antagonism toward the intestinal microbiota and its effect on Vibrio cholerae virulence. Science 359:210-213.

19. Miyata ST, Unterweger D, Rudko SP, Pukatzki S. 2013. Dual Expression Profile of Type VI Secretion System Immunity Genes Protects Pandemic Vibrio cholerae. PLoS Pathog 9:e1003752.

20. Miyata ST, Kitaoka M, Brooks TM, McAuley SB, Pukatzki S. 2011. Vibrio cholerae Requires the Type VI Secretion System Virulence Factor VasX To Kill Dictyostelium discoideum. Infect Immun 79:2941-2949.

21. Russell AB, LeRoux M, Hathazi K, Agnello DM, Ishikawa T, Wiggins PA, Wai SN, Mougous JD. 2013. Diverse type VI secretion phospholipases are functionally plastic antibacterial effectors. Nature 496:508-512.

22. Ho BT, Fu Y, Dong TG, Mekalanos JJ. 2017. Vibrio cholerae type 6 secretion system effector trafficking in target bacterial cells. Proc Natl Acad Sci 114:9427-9432.

23. Brooks TM, Unterweger D, Bachmann V, Kostiuk B, Pukatzki S. 2013. Lytic Activity of 
the Vibrio cholerae Type VI Secretion Toxin VgrG-3 Is Inhibited by the Antitoxin TsaB. J Biol Chem 288:7618-7625.

24. Crisan C V., Hammer BK. 2020. The Vibrio cholerae type VI secretion system: toxins, regulators and consequences. Environ Microbiol 22:4112-4122.

25. Crisan C V., Chande AT, Williams K, Raghuram V, Rishishwar L, Steinbach G, Watve SS, Yunker P, Jordan IK, Hammer BK. 2019. Analysis of Vibrio cholerae genomes identifies new type VI secretion system gene clusters. Genome Biol 20:163.

26. Unterweger D, Miyata ST, Bachmann V, Brooks TM, Mullins T, Kostiuk B, Provenzano D, Pukatzki S. 2014. The Vibrio cholerae type VI secretion system employs diverse effector modules for intraspecific competition. Nat Commun 5:3549.

27. Altindis E, Dong T, Catalano C, Mekalanos J. 2015. Secretome Analysis of Vibrio cholerae Type VI Secretion System Reveals a New Effector-Immunity Pair. MBio 6:e00075.

28. Hersch SJ, Watanabe N, Stietz MS, Manera K, Kamal F, Burkinshaw B, Lam L, Pun A, Li M, Savchenko A, Dong TG. 2020. Envelope stress responses defend against type six secretion system attacks independently of immunity proteins. Nat Microbiol 5:706-714.

29. Santoriello FJ, Michel L, Unterweger D, Pukatzki S. 2020. Pandemic Vibrio cholerae shuts down site-specific recombination to retain an interbacterial defence mechanism. Nat Commun 11:6246.

30. Kirchberger PC, Unterweger D, Provenzano D, Pukatzki S, Boucher Y. 2017. Sequential displacement of Type VI Secretion System effector genes leads to evolution of diverse immunity gene arrays in Vibrio cholerae. Sci Rep 7:45133.

31. Drebes Dörr NC, Blokesch M. 2020. Interbacterial competition and anti-predatory behaviour of environmental Vibrio cholerae strains. Environ Microbiol 22:4485-4504.

32. Labbate M, Orata FD, Petty NK, Jayatilleke ND, King WL, Kirchberger PC, Allen C, Mann G, Mutreja A, Thomson NR, Boucher Y, Charles IG. 2016. A genomic island in Vibrio cholerae with VPI-1 site-specific recombination characteristics contains CRISPRCas and type VI secretion modules. Sci Rep 6:36891.

33. Cascales E, Cambillau C. 2012. Structural biology of type VI secretion systems. Philos Trans R Soc Lond B Biol Sci 367:1102-1111.

34. Unterweger D, Kostiuk B, Ötjengerdes R, Wilton A, Diaz-Satizabal L, Pukatzki S. 2015. Chimeric adaptor proteins translocate diverse type VI secretion system effectors in Vibrio cholerae. EMBO J 34:2198-2210.

35. Shneider MM, Buth SA, Ho BT, Basler M, Mekalanos JJ, Leiman PG. 2013. PAARrepeat proteins sharpen and diversify the type VI secretion system spike. Nature 500:350353.

36. Durand E, Derrez E, Audoly G, Spinelli S, Ortiz-Lombardia M, Raoult D, Cascales E, Cambillau C. 2012. Crystal Structure of the VgrG1 Actin Cross-linking Domain of the Vibrio cholerae Type VI Secretion System. J Biol Chem 287:38190-38199. 
37. Ma AT, Mekalanos JJ. 2010. In vivo actin cross-linking induced by Vibrio cholerae type VI secretion system is associated with intestinal inflammation. Proc Natl Acad Sci 107:4365-4370.

38. Karaolis DKR, Johnson JA, Bailey CC, Boedeker EC, Kaper JB, Reeves PR. 1998. A Vibrio cholerae pathogenicity island associated with epidemic and pandemic strains. Proc Natl Acad Sci U S A 95:3134-3139.

39. Watve SS, Thomas J, Hammer BK. 2015. CytR Is a Global Positive Regulator of Competence, Type VI Secretion, and Chitinases in Vibrio cholerae. PLoS One 10:e0138834.

40. Lo Scrudato M, Blokesch M. 2013. A transcriptional regulator linking quorum sensing and chitin induction to render Vibrio cholerae naturally transformable. Nucleic Acids Res 41:3644-3658.

41. Jaskolska M, Stutzmann S, Stoudmann C, Blokesch M. 2018. QstR-dependent regulation of natural competence and type VI secretion in Vibrio cholerae. Nucleic Acids Res 46:10619-10634.

42. Dong TG, Ho BT, Yoder-Himes DR, Mekalanos JJ. 2013. Identification of T6SSdependent effector and immunity proteins by Tn-seq in Vibrio cholerae. Proc Natl Acad Sci 110:2623-2628.

43. Liang X, Kamal F, Pei T, Xu P, Mekalanos JJ, Dong TG. 2019. An onboard checking mechanism ensures effector delivery of the type VI secretion system in Vibrio cholerae. Proc Natl Acad Sci 116:23292-23298.

44. Crisan C V, Nichols HL, Wiesenfeld S, Steinbach G, Yunker PJ, Hammer BK. 2021. Glucose confers protection to Escherichia coli against contact killing by Vibrio cholerae. Sci Rep 11:2935.

45. Almagro Armenteros JJ, Tsirigos KD, Sønderby CK, Petersen TN, Winther O, Brunak S, von Heijne G, Nielsen H. 2019. SignalP 5.0 improves signal peptide predictions using deep neural networks. Nat Biotechnol 37:420-423.

46. Krogh A, Larsson B, von Heijne G, Sonnhammer EL. 2001. Predicting transmembrane protein topology with a hidden markov model: application to complete genomes. J Mol Biol 305:567-580.

47. Käll L, Krogh A, Sonnhammer ELL. 2004. A Combined Transmembrane Topology and Signal Peptide Prediction Method. J Mol Biol 338:1027-1036.

48. Drozdetskiy A, Cole C, Procter J, Barton GJ. 2015. JPred4: a protein secondary structure prediction server. Nucleic Acids Res 43:W389-W394.

49. Samsudin F, Ortiz-Suarez ML, Piggot TJ, Bond PJ, Khalid S. 2016. OmpA: A Flexible Clamp for Bacterial Cell Wall Attachment. Structure 24:2227-2235.

50. Grizot S, Buchanan SK. 2004. Structure of the OmpA-like domain of RmpM from Neisseria meningitidis. Mol Microbiol 51:1027-1037. 
51. De Mot R, Proost P, Van Damme J, Vanderleyden J. 1992. Homology of the root adhesin of Pseudomonas fluorescens $\mathrm{OE} 28.3$ with porin $\mathrm{F}$ of $P$. aeruginosa and $P$. syringae. Mol Gen Genet MGG 231:489-493.

52. Smith SGJ, Mahon V, Lambert MA, Fagan RP. 2007. A molecular Swiss army knife: OmpA structure, function and expression. FEMS Microbiol Lett 273:1-11.

53. LaCourse KD, Peterson SB, Kulasekara HD, Radey MC, Kim J, Mougous JD. 2018. Conditional toxicity and synergy drive diversity among antibacterial effectors. Nat Microbiol 3:440-446.

54. Mariano G, Trunk K, Williams DJ, Monlezun L, Strahl H, Pitt SJ, Coulthurst SJ. 2019. A family of Type VI secretion system effector proteins that form ion-selective pores. Nat Commun 10.

55. Sánchez E, García S, Heredia N. 2010. Extracts of edible and medicinal plants damage membranes of Vibrio cholerae. Appl Environ Microbiol 76:6888-6894.

56. Ji X, Zou J, Peng H, Stolle A-S, Xie R, Zhang H, Peng B, Mekalanos JJ, Zheng J. 2019. Alarmone Ap4A is elevated by aminoglycoside antibiotics and enhances their bactericidal activity. Proc Natl Acad Sci 116:9578 LP - 9585.

57. Finn RD, Clements J, Eddy SR. 2011. HMMER web server: interactive sequence similarity searching. Nucleic Acids Res 39:W29-W37.

58. Fridman CM, Keppel K, Gerlic M, Bosis E, Salomon D. 2020. A comparative genomics methodology reveals a widespread family of membrane-disrupting T6SS effectors. Nat Commun 11.

59. Benarroch JM, Asally M. 2020. The Microbiologist's Guide to Membrane Potential Dynamics. Trends Microbiol 28:304-314.

60. Maloney PC, Kashket ER, Wilson TH. 1974. A protonmotive force drives ATP synthesis in bacteria. Proc Natl Acad Sci U S A 71:3896-3900.

61. Strahl H, Hamoen LW. 2010. Membrane potential is important for bacterial cell division. Proc Natl Acad Sci 107:12281 LP - 12286.

62. Peraro MD, Van Der Goot FG. 2016. Pore-forming toxins: Ancient, but never really out of fashion. Nat Rev Microbiol 14:77-92.

63. Hinds MG, Zhang W, Anderluh G, Hansen PE, Norton RS. 2002. Solution structure of the eukaryotic pore-forming cytolysin equinatoxin II: implications for pore formation. J Mol Biol 315:1219-1229.

64. Cascales E, Buchanan SK, Duché D, Kleanthous C, Lloubès R, Postle K, Riley M, Slatin S, Cavard D. 2007. Colicin Biology. Microbiol Mol Biol Rev 71:158-229.

65. Yamashita K, Kawai Y, Tanaka Y, Hirano N, Kaneko J, Tomita N, Ohta M, Kamio Y, Yao M, Tanaka I. 2011. Crystal structure of the octameric pore of staphylococcal $\gamma$ hemolysin reveals the $\beta$-barrel pore formation mechanism by two components. Proc Natl Acad Sci U S A 108:17314-17319. 
590

591

592

593

594

595

596

597

66. Savva CG, Da Costa SPF, Bokori-Brown M, Naylor CE, Cole AR, Moss DS, Titball RW, Basak AK. 2013. Molecular architecture and functional analysis of NetB, a pore-forming toxin from Clostridium perfringens. J Biol Chem 288:3512-3522.

67. Mueller M, Grauschopf U, Maier T, Glockshuber R, Ban N. 2009. The structure of a cytolytic $\alpha$-helical toxin pore reveals its assembly mechanism. Nature 459:726-730.

68. Song L, Hobaugh MR, Shustak C, Cheley S, Bayley H, Gouaux JE. 1996. Structure of Staphylococcal $\alpha$-Hemolysin, a Heptameric Transmembrane Pore. Science 274:1859 LP 1865.

69. Maharjan S, Saleem M, Feavers IM, Wheeler JX, Care R, Derrick JP. 2016. Dissection of the function of the RmpM periplasmic protein from Neisseria meningitidis. Microbiol $162: 364-375$.

70. Yoh M, Matsuyama J, Ohnishi M, Takagi K, Miyagi H, Mori K, Park KS, Ono T, Honda T. 2005. Importance of Providencia species as a major cause of travellers' diarrhoea. J Med Microbiol 54:1077-1082.

71. Endimiani A, Luzzaro F, Brigante G, Perilli M, Lombardi G, Amicosante G, Rossolini GM, Toniolo A. 2005. Proteus mirabilis bloodstream infections: Risk factors and treatment outcome related to the expression of extended-spectrum $\beta$-lactamases. Antimicrob Agents Chemother 49:2598-2605.

72. Dzeing-Ella A, Szwebel TA, Loubinoux J, Coignard S, Bouvet A, Le Jeunne C, Aslangul E. 2009. Infective endocarditis due to Citrobacter koseri in an immunocompetent adult. J Clin Microbiol 47:4185-4186.

73. Abbott SL, Janda JM. 1994. Isolation of Yokenella regensburgei ('Koserella trabulsii') from a patient with transient bacteremia and from a patient with a septic knee. J Clin Microbiol 32:2854-2855.

74. Di Martino ML, Ek V, Hardt WD, Eriksson J, Sellin ME. 2019. Barcoded consortium infections resolve cell type-dependent Salmonella enterica serovar Typhimurium entry mechanisms. MBio 10:1-15.

75. Frans I, Michiels CW, Bossier P, Willems KA, Lievens B, Rediers H. 2011. Vibrio anguillarum as a fish pathogen: virulence factors, diagnosis and prevention. J Fish Dis 34:643-661.

76. Ciche TA, Ensign JC. 2003. For the insect pathogen Photorhabdus luminescens, which end of a nematode is out? Appl Environ Microbiol 69:1890-1897.

77. Bellieny-Rabelo D, Tanui CK, Miguel N, Kwenda S, Shyntum DY, Moleleki LN. 2019. Transcriptome and Comparative Genomics Analyses Reveal New Functional Insights on Key Determinants of Pathogenesis and Interbacterial Competition in Pectobacterium and Dickeya spp. Appl Environ Microbiol 85:1-20.

78. Russell AB, Hood RD, Bui NK, LeRoux M, Vollmer W, Mougous JD. 2011. Type VI secretion delivers bacteriolytic effectors to target cells. Nature 475:343-347.

79. Skorupski K, Taylor RK. 1996. Positive selection vectors for allelic exchange. Gene 
605

606

607

608

609

610

611

612

613

614

615

616

617

618

619

620

621

622

623

624

625

626

627

628

629

630

631

632

633

635

169:47-52.

80. Marvig RL, Blokesch M. 2010. Natural transformation of Vibrio cholerae as a tool Optimizing the procedure. BMC Microbiol 10:155.

81. Watve SS, Bernardy EE, Hammer BK. 2014. Vibrio cholerae: Measuring natural transformation frequency. Curr Protoc Microbiol 2014:6A.4.1-6A.4.12.

82. HMMER: biosequence analysis using profile hidden Markov modelsv. 3.1b2.

83. Edgar RC. 2004. MUSCLE: Multiple sequence alignment with high accuracy and high throughput. Nucleic Acids Res 32:1792-1797.

84. Guindon S, Dufayard J-F, Lefort V, Anisimova M, Hordijk W, Gascuel O. 2010. New Algorithms and Methods to Estimate Maximum-Likelihood Phylogenies: Assessing the Performance of PhyML 3.0. Syst Biol 59:307-321.

85. Lefort V, Longueville JE, Gascuel O. 2017. SMS: Smart Model Selection in PhyML. Mol Biol Evol 34:2422-2424.

86. Lemoine F, Correia D, Lefort V, Doppelt-Azeroual O, Mareuil F, Cohen-Boulakia S, Gascuel O. 2019. NGPhylogeny.fr: New generation phylogenetic services for nonspecialists. Nucleic Acids Res 47:W260-W265.

\section{ACKNOWLEDGEMENTS}

We thank Dr. Jacob Thomas, Dr. Mackenzie Martin, Dr. Athéna Patterson-Orazem, Dr. Dustin Huard and Tong Yu for advice, experimental help and useful discussions.

BKH would also like to thank funding from the Georgia Institute of Technology School of Biological Sciences, NSF (MCB 1149925 and BMAT-2003721) and BSF (2015103). GS would like to thank the German National Academy of Natural Sciences Leopoldina (LDPS 2017-03).

\section{COMPETING INTERESTS}

The authors declare no competing interests.

\section{SUPPLEMENTARY FILES LEGENDS}

Supplementary Figure 1. TpeV Phobius transmembrane helix prediction.

\section{Supplementary Figure 2. TpeV SignalP prediction.}


636 Supplementary Table 1. Complete list of all identified TpeV homologs using the PHMMER

637 algorithm.

638 Supplementary Table 2. Strains and plasmids used in this study. 